\title{
FINAL REPORT DOE GRANT
}

DE-FG02-00ER15074 (Matthysse) and DE-FG03-00ER15073 (White)

\section{General introduction}

This research project is collaboration between the laboratories of Ann Matthysse at the University of North Carolina at Chapel Hill and Alan White at North Dakota State University. Dr. Matthysse's expertise is in bacterial genetics and bacterial adhesion; Dr. White's expertise is in carbohydrate biochemistry. We received this grant in August 2000. It represents a new collaboration for both of us, as we had not collaborated since 1994 when we showed that the polysaccharide made by Agrobacterium tumefaciens was cellulose. The research was started from work in our two labs that was only indirectly related to this project. Some time was required to hire personnel (both labs) and to purchase equipment (White-NDSU) so that we actually began these studies in October 2000. This report represents the work done between that date and July 2004.

A short summary of our present results is as follows: we have cloned the celC gene and its homologue from E. coli, yhjM, in an expression vector and expressed the both genes in E. coli; we have determined that the YhjM protein is able to complement in vitro cellulose synthesis by extracts of $A$. tumefaciens celC mutants, we have purified the YhjM protein product and are currently examining its enzymatic activity; we have examined whole cell extracts of CelC and various other cellulose mutants and wild type bacteria for the presence of cellulose oligomers and cellulose; we have examined the ability of extracts of wild type and cellulose mutants including CelC to incorporate UDP- ${ }^{14} \mathrm{C}$-glucose into cellulose and into water-soluble, ethanol-insoluble oligosaccharides; we have made mutants which synthesize greater amounts of cellulose than the wild type; and we have examined the role of cellulose in the formation of biofilms by A. tumefaciens. In addition we have examined the ability of a putative cellulose synthase gene from the tunicate Ciona savignyi to complement an A.tumefaciens celA mutant. The greatest difference between our knowledge of bacterial cellulose synthesis when we started this project and current knowledge is that in 1999 when we wrote the original grant very few bacteria were known to synthesize cellulose and genes involved in this synthesis were sequenced only from Acetobacter species, A. tumefaciens and Rhizobium leguminosarum. Currently many bacteria are known to synthesize cellulose and genes that may be involved have been sequenced from more than 10 species of bacteria. This additional information has raised the possibility of attempting to use genes from one bacterium to complement mutants in another bacterium. This will enable us to examine the question of which genes are responsible for the three dimensional structure of cellulose (since this differs among bacterial species) and also to examine the interactions between the various proteins required for cellulose synthesis. We have carried out one preliminary experiment of this type and have successfully complemented an A. tumefaciens CelC mutant with the homologous gene (yhjM) from E. coli.

\section{Bacterial genes required for cellulose synthesis}

Cellulose is made by higher plants and by a number of bacteria as well. The study of the production of cellulose by bacteria has the advantage that mutants are relatively easy to make and analyze and that the complete genomes of several bacteria that are capable of producing cellulose have been sequenced. These include Agrobacterium tumefaciens, Aquifex aeolicus, Escherichia coli, Salmonella typhi, S. typhimurium and Xanthomonas citri. In addition genes required for cellulose synthesis have been sequenced in Acetobacter xylinum (several strains), Pseudomonas fluorescens, and Rhizobium leguminosarum. In A. tumefaciens we have previously used transposon mutagenesis to identify genes required for cellulose synthesis. A. tumefaciens contains a circular chromosome, a linear chromosome, and two plasmids (pTi and pAT). The cel genes are located on the linear chromosome. Genes required for cellulose synthesis include celA, celB, celC, and celE (Fig. 1).

CelA is the most conserved of the cellulose synthesis proteins. It has homology to the cellulose synthase (BcsA) of A. xylinum that has been shown to bind UDP-glucose (Saxena, Lin, and Brown, 1990). 
The UDP-glucose binding site is retained in the A. tumefaciens CelA protein. All of the organisms known to synthesize cellulose whose genomes have been sequenced (including higher plants) contain genes encoding one or more homologues to the CelA protein.

CelC is moderately conserved. It has homology to type 8 glycohydrolases including endoglucanases from Erwinia rapontici and E. chrysamthemi. All of the sequenced bacterial cellulose synthesis regions contain a gene for a protein homologous to CelC. The celC gene is required for cellulose synthesis in A. tumefaciens(Matthysse, White, and Lightfoot, 1995), A. xylinum(Standal et al., 1994) and S. enteriditis(Zogaj et al., 2003)(other bacteria have not been tested). The function of this protein in cellulose synthesis is a major focus of our studies.

Homologues to CelB appear to split into 2 groups: one containing the genes from A. tumefaciens and $R$. leguminosarum and the other containing the genes from A. xylinum, E. coli, P. fluorescens, $S$. typhi, $S$. typhimurium and $X$. citri. The gene from $A$. aeolicus is distantly related to both groups as might be expected from the position of this organism on the evolutionary tree of the bacteria near the bottom of the eubacteria just above the split between eubacteria and archaea. CelB from any bacterium has no homology to any protein known to have enzymatic activity nor does it share any conserved motifs with other proteins. CelB is required for cellulose synthesis in all organisms in which it has been tested. The role of CelB in cellulose synthesis is not understood. It has been proposed that in A. xylinum it may bind cyclic diguanlyic acid to regulate cellulose synthesis (Weinhouse et al., 1997).

CelE is homologous to proteins that split C-N bonds including hippurate hydrolase and carboxypeptidase. A gene with high homology to celE is located immediately next to the operon containing celA in R. leguminosarum (Ausmees et al., 1999). In E. coli, S. typhi, S. typhimurium, and X. citri a gene with homology to peptidases (but no direct homology to A. tumefaciens celE is located adjacent to the operon containing the cellulose synthase. In A. tumefaciens and S. enteriditis celE (bcsE) has been shown to be required for cellulose synthesis(Matthysse, White, and Lightfoot, 1995;Romling et al., 2003)

Two other genes are found in the cel region in A. tumefaciens: celG which is between celE and celC oriented in the same direction as celC and celD which is adjacent to celE. However, we currently have no evidence to show that these genes are required for cellulose synthesis. CelD has no homologues in those bacteria with sequenced genomes that synthesize cellulose including A. aeolicus, E. coli, S. typhi, $S$. typhimurium and $X$. citri. At present the only mutants we have in celD are transposon insertions that are polar and would prevent expression of celE as well as celD. As expected these insertions block cellulose synthesis since bacteria carrying insertions in celE are unable to synthesize cellulose. The CelG protein has no homologues at all in the database. We made an insertion in celG and found, unexpectedly, that bacteria carrying this mutation produce more cellulose that the parent strain. One other gene involved in the regulation of cellulose synthesis has been identified, celR. This gene is located on the linear chromosome some distance from the cel genes. It has homology to helix-turn-helix DNA binding proteins. Insertions in celR cause the bacteria to overproduce cellulose.

\section{The role of CelC in cellulose synthesis:}

Carbohydrate composition of the CelC mutant as compared to the wild type parent

We have found that CelC mutants grow poorly when compared with the parent strain. Spectra of the A1045 wild type, the CelC mutant strain, and the subtracted difference between the two strains (difference) are shown in Figure 2. The bacteria were collected, washed, freeze-dried, and analyzed. Both the wild type and the CelC mutant show the amide stretching region of proteins at 1650 and $1550 \mathrm{~cm}^{-1}$ and a carboxylate ion peak at $1414 \mathrm{~cm}^{-1}$. However, the carbohydrate fingerprint region between 800 and 1000 $\mathrm{cm}^{-1}$ indicates a difference in the properties of the CelC mutant, relative to the wild type, which can clearly be seen in the spectra of the subtracted difference between the two strains. Peaks at 897 and $945 \mathrm{~cm}-1$ clearly indicate a glycosidic linkage in the CelC mutant not present in the wild type. This 'difference' spectrum also indicates an alteration in the $1700 \mathrm{~cm}^{-1}$ region of the CelC mutant, relative to the wild type. 
Incorporation of UDP- ${ }^{14} \mathrm{C}$-glucose by extracts of the CelC mutant and wild type parent strains: the nature of the products

When extracts of wild type $A$. tumefaciens are incubated with UDP- ${ }^{14} \mathrm{C}$-glucose they incorporate ${ }^{14} \mathrm{C}$-glucose into cellulose. Extracts of the CelC mutant fail to synthesize cellulose. However, they do incorporate UDP- ${ }^{14} \mathrm{C}$-glucose into oligosaccharides that are soluble in water and can be precipitated by $80 \%$ ethanol. These oligosaccharides are incorporated into cellulose by extracts of the wild type bacteria. Capillary electrophoresis was used to determine the structure of the product of the CelC mutant strain. Figure 3 shows the retention times for radioactive products made by extracts of wild type and the CelC mutant as well as differences in retention times following endoglucanase II (EG-II) digestion. (This enzyme is specific for $\beta$-(1,4) linked glucose residues). Cello-oligosaccharide standards ranging from 2 to 5 glucose moieties are shown for comparison (top trace). The A1045 wild-type extract contained glucose moieties no bigger than 3 units long and appeared to be unaffected by EG-II digestion. This may be due to the inability of the enzyme to cleave glucose units close to the APTS, which was used to make a derivative on the reducing end in this system. This strain may also contain sugar units that are not $\beta$ - $(1,4)$-linked. The CelC mutant contained sugar units that have different retention times than $\beta$-(1,4)-linked $\mathrm{Glu}_{3}$, Glu 4 and $\mathrm{Glu}_{5}$. This can be clearly seen when the sample was 'spiked' with the cello-oligosaccharide standards. Furthermore, these novel short chain moieties appear to contain some $\beta$ - $(1,4)$-linkages as they disappeared following EG-II digestion (bottom trace). This reduction in the longer chain oligosaccharides was accompanied by a concomitant increase in the $\mathrm{Glu}_{3}$ peak (B).

\section{The presence of $\beta-(1,3)$-linked glucans in the CelC mutant intermediates}

A combination of capillary electrophoresis (C.E.) and methylation analysis was used to further investigate the oligosaccharides containing glucose present in the A1045 wild-type and the CelC mutant. Laminarin standards ranging from $\mathrm{Glc}_{2}$ to Glc $_{5}$ were derivatized with APTS and analyzed. These laminarin standards are comprised of $\beta$-(1,3)-linked glucans. When compared with $\beta$-(1,4)-linked glucan standards (Glc to Glc $_{5}$ ) the relative retention times of the $\beta$-(1,3)-linked glucans were offset by an additional two minutes (Fig.4A). This factor of increased retention time may be responsible for the altered retention time seen in the $\mathrm{CE}$ analysis of the accumulated glucan intermediates of the CelC mutant. Methylation analysis on the glucans from CelC mutant and the A1045 wild-type by GC-FID provides more conclusive evidence for the presence of $\beta$ - $(1,3)$-linked glucans in both of these samples. A small proportion ( $6 \mathrm{~mol} \%$ of the total glucose) of $(1,3)$-linked glucans was present in the wild-type, which may account for the increased retention time of the Glc 3 peak seen by CE in this sample. This was expected as A. tumefaciens makes a $\beta$-(1,3)-linked glucose polymer called curdlan. The enzymes for this are separate from the cellulose synthesis enzymes and are located on the circular chromosome (Stasinopoulos et al., 1999).

The CelC mutant glucose-containing oligosaccharides contained a comparatively high proportion of $(1,3)$-linked glucan (37 mol\% of the total glucose present in the mutant) relative to the wild-type sample. When these intermediates were subjected to enzymatic digestion by a $\beta-(1,3)$ laminarinase the novel short chain moieties disappeared. These data suggest that the oligosaccharides that accumulate in the CelC mutant contain a mixture of $\beta-(1,3)$ - and $\beta-(1,4)$ - linked glucans.

Identification of a potential lipid-linked intermediate

It is possible that the oligosaccharides that accumulate in the CelC mutant are lipid-linked since they can be extracted with chloroform-methanol (Matthysse, Thomas, and White, 1995). In order to determine the nature of this lipid intermediate, we carried out a standard lipid extraction first used by Ohana et al (X). Briefly, 1 liter of culture of the wild type and the CelC mutant were dried down and homogenized in boiling isopropanol and filtered through Miracloth. The insoluble residue was reextracted with water and re-filtered. Both filtrates were combined, precipitated in $80 \%$ ethanol overnight and centrifuged. The soluble material was concentrated under air. Recently, it has been suggested that this intermediate is an allylically linked glucosyl-1-phospholipid (Chilton and Matthysse, personal 
communication) and its structure is shown in Figure 5. We believe that the lipid is undecaprenol $\left(\mathrm{C}_{55} \mathrm{H}_{90} \mathrm{O}\right)$. We are currently determining the most appropriate method to visualize and identify the isolated lipids from the wild type and CelC mutant.

\section{Cloning, expression, and purification of the CelC protein}

The celC gene was cloned using PCR into the expression vector pPROTet.E133 (Clontech). This vector has an Ltet $0-1$ promoter whose activity can be regulated by anhydrotetracycline (this compound does not have antibiotic activity against $E$. coli). The multiple cloning site in the vector is preceded by a six histidines (in frame) and a site for cleavage by enterokinase. The protein was purified using a nickel resin to which it binds due to the his-tag at its beginning. The activity of the purified protein is currently under investigation. Unfortunately this clone is very poorly tolerated by E. coli and it was difficult to obtain much protein. In addition the imiazole required to elute the histidine-linked cloned protein from the resin inhibits cellulose synthesis. We attempted to overcome these problems by cloning the $E$. coli celC equivalent, $y$ hjM, in a vector (pCalN) which results in the production of a calmodulin-linked protein. This cloned protein can be purified on a calcium-containing resin and eluted with EGTA. Low concentrations of EGTA do not interfere with in vitro cellulose synthesis. Using this system we have purified a protein which is able to complement extracts of the celC mutant resulting in cellulose synthesis in vitro. We are currently determining how to stabilize the protein for shipping to North Dakota for analysis of its interactions with oligosaccharides.

Complementation of CelC mutants

A clone carrying the celC gene behind a constitutive promoter was constructed in a shuttle vector (pBBRmcs-5) and introduced from E. coli into A. tumefaciens. This vector is low copy number plasmid present in 5-10 copies per bacterium. The clone was able to complement the CelC mutation in vivo and allowed the mutant to grow at a normal rate. The complemented mutant no longer accumulated the oligosaccharides found in the uncomplemented CelC mutant. It made some cellulose in vivo but extracts of the complemented mutant synthesized very little cellulose in vitro. One possible interpretation of this result is that the ratio of the various Cel proteins to each other may be important for normal cellulose synthesis.

In order to compare the functions of CelC homologues from various cellulose-synthesizing bacteria, the homologous gene, yhjM, was cloned from $E$. coli and introduced into A. tumefaciens using the same shuttle vector and promoter as was used for the $A$. tumefaciens celC gene. The results were the same as those observed with the A. tumefaciens gene suggesting that the E. coli yhjM gene can substitute for celC.

\section{Regulation of cellulose synthesis in A. tumefaciens}

Previous work had identified a Tn5 insertion that resulted in overproduction of cellulose by $A$. tumefaciens. The insertion was cloned and the region surrounding it was sequenced. A gene (celR) was identified which has homology to DNA binding proteins. Insertions were made in celR in wild type and CelA and CelB mutant bacteria. In the wild type background the insertion led to overproduction of cellulose. The CelR mutation had no apparent effect in the CelA or CelB mutant background. We attempted to construct a CelR mutant in the CelC mutant background but the resulting bacteria grew very poorly and died after a few transfers. The toxicity of this mutation in the CelC background may be due to over-accumulation of the lipid-linked oligosaccharides that we have observed in the CelC mutant.

As mentioned above we found that an insertion in the celG gene resulted in bacteria that overproduced cellulose. The mechanism underlying this effect is not understood.

\section{The role of cellulose in bacterial adherence and biofilm formation}

Cellulose-minus and cellulose-overproducing mutants were used to explore the role of cellulose in the adherence of $A$. tumefaciens to plant (particularly root) surfaces and in biofilm formation on root and plastic surfaces. The wild type bacteria bind well to roots and form a biofilm on both roots and plastic. 
Cellulose-minus mutants show very little binding to roots and do not form a biofilm on root or plastic surfaces. CelR mutants showed increased binding to tomato roots. Microscopic observations showed that they formed a deeper layer of bacterial biofilm on the root than the parent strain. Overproduction of cellulose due to mutations in the celR or celG genes allowed A. tumefaciens mutant strains A1045 and A205 (which ordinarily do not bind to or form a biofilm on tomato roots (Matthysse and McMahan, 1998) ) to bind and form a biofilm (see Figure 6).

Identification of the cellulose synthase gene from a tunicate In collaboration with William Smith (UCSB) the cellulose synthase gene from $C$. savignyi (a tunicate) was cloned and the predicted protein found to be related to A. tumefaciens CelA(Matthysse et al., 2004). A cDNA clone of the tunicate gene could complement an Agrobacterium celA mutant for cellulose synthesis both in vivo and in vitro. In addition, the tunicate gene included a region near the $\mathrm{C}$ terminal end with homology to endoglucanases of a different family than CelC. A clone of this region of the tunicate cDNA was able to complement an Agrobacterium celC mutant. The closest relatives to the tunicate cellulose synthase gene as judged by are all bacterial cellulose synthase genes. No homologues are found in any animal sequences currently available in the data bases. Thus the tunicate may have acquired its cellulose synthase gene by lateral transfer from a bacterium.

\section{Publications}

Matthysse, A.G. 2001. Bacterial cellulose. In: Encyclopedia of Plant Pathology. O. Malloy and T. Murray, eds. John Wiley \& Sons, Inc. 777-778.

Marry, Mazz, Matthysse, Ann G. and White, Alan R. 2001. The role of the celC gene product in cellulose synthesis by A. tumefaciens. Ninth International Cell Wall Conference.

Marry, Mazz, Matthysse, Ann G. and White, Alan R. 2002. The role of the celC gene product in cellulose synthesis by Agrobacterium tumefaciens. Plant cell wall biosynthesis meeting.

Marry, Mazz, White, Alan R. and Ann G. Matthysse. 2001. Mechanism of cellulose synthesis by Agrobacterium tumefaciens. $22^{\text {nd }}$ Crown Gall Conference. Abstract.

Marry, Mazz., Matthysse, Ann G. and White, Alan R.. Characterization of oligosaccharides which accumulate in CelC mutants of Agrobacterium tumefaciens. (Manuscript in preparation).

A. G. Matthysse, M. Marry, L. Krall, M. Kaye, BE Ramey, C. Fuqua, and A. R. White. The effect of cellulose overproduction on binding and biofilm formation on roots by Agrobacterium tumefaciens. In preparation., 2004.

A. G. Matthysse, K. Deschet, M. Williams, M. Marry, A. R. White, and W. C. Smith. A functional cellulose synthase from ascidian epidermis. Proc.Natl.Acad.Sci.U.S.A 101 (4):986-991, 2004.

Bronwyn E. Ramey, Ann G. Matthysse, and Clay Fuqua. The FNR-type transcriptional regulator SinR controls maturation of Agrobacterium tumefaciens biofilms. Molecular Microbiology 52 (5):1495-1511, 2004. 


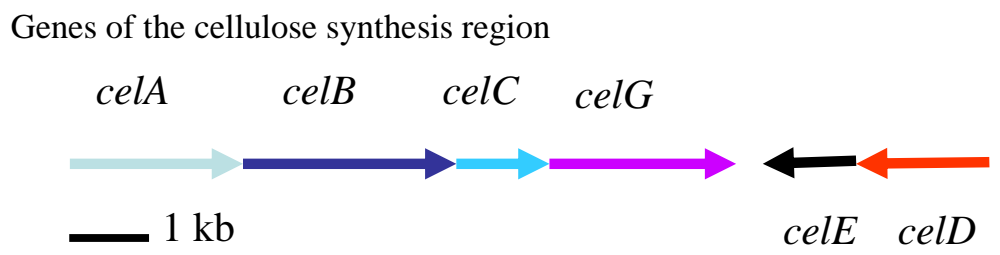

Figure 1. Genes of the cellulose synthesis region of Agrobacterium tumefaciens. The genes are located on the linear chromosome. The arrows indicate the direction of the open reading frames. 


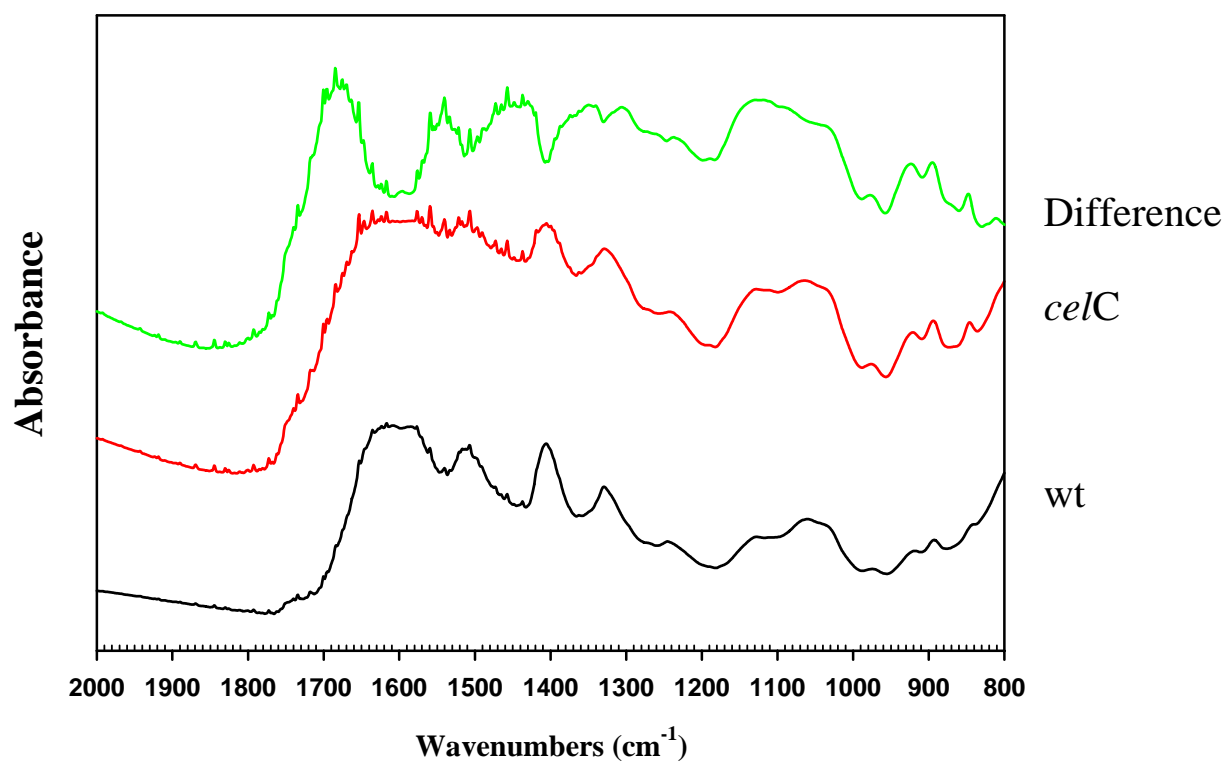

Figure 2: FT-IR spectra of the celC mutant and the A1045 wild type strain. Both the wild type and the CelC mutant show the amide stretching region of proteins at 1650 and $1550 \mathrm{~cm}^{-1}$ and a carboxylate ion peak at $1414 \mathrm{~cm}^{-1}$. However, the carbohydrate fingerprint region between 800 and $1000 \mathrm{~cm}^{-1}$ indicates a difference in the properties of the CelC mutant, relative to the wild type, which can clearly be seen in the spectra of the subtracted difference between the two strains. Peaks at 897 and $945 \mathrm{~cm}-1$ clearly indicate a glycosidic linkage in the CelC mutant not present in the wild type. This 'difference' spectrum also indicates an alteration in the $1700 \mathrm{~cm}^{-1}$ region of the CelC mutant, relative to the wild type. 


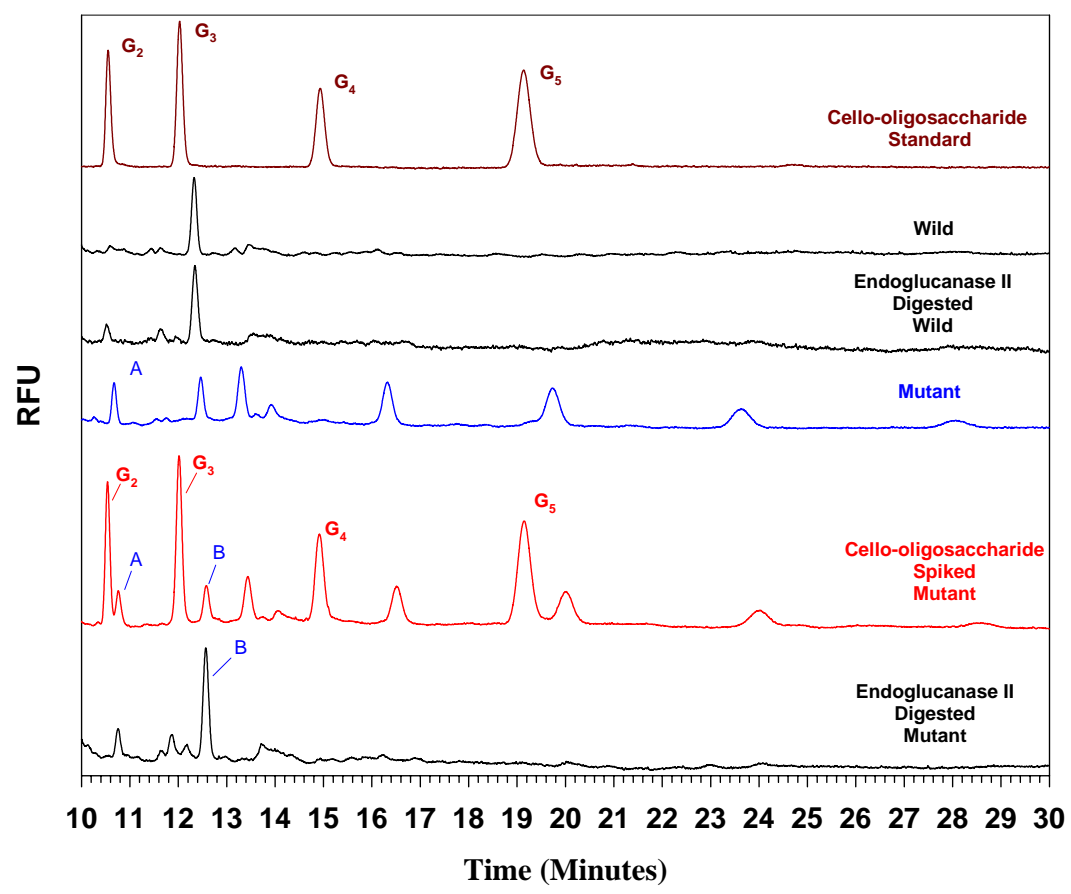

Figure 3: Capillary electrophoresis retention time analysis of the A1045 'wild type' and the celC mutant, as well as differences in retention times following endoglucanase II (EG-II) digestion. Cellooligosaccharide standards ranging from 2 to 5 glucose moieties are shown for comparison (top trace). The A1045 wild type extract contained glucose moieties no bigger than 3 units long and appeared to be unaffected by EG-II digestion. This may be due to the inability of the enzyme to cleave glucose units close to the APTS, which was used to make a derivative on the reducing end in this system. This strain may also contain sugar units that are not $\beta$ - $(1,4)$-linked. The CelC mutant contained sugar units that have different retention times than $\beta$-(1,4)-linked $\mathrm{Glu}_{3}, \mathrm{Glu}_{4}$ and $\mathrm{Glu}_{5}$. This can be clearly seen when the sample was 'spiked' with the cello-oligosaccharide standards. Furthermore, these novel short chain moieties appear to contain some $\beta$-(1,4)-linkages as they disappeared following EG-II digestion (bottom trace). This reduction in the longer chain oligosaccharides was accompanied by a concomitant increase in the $\mathrm{Glu}_{3}$ peak (B). 


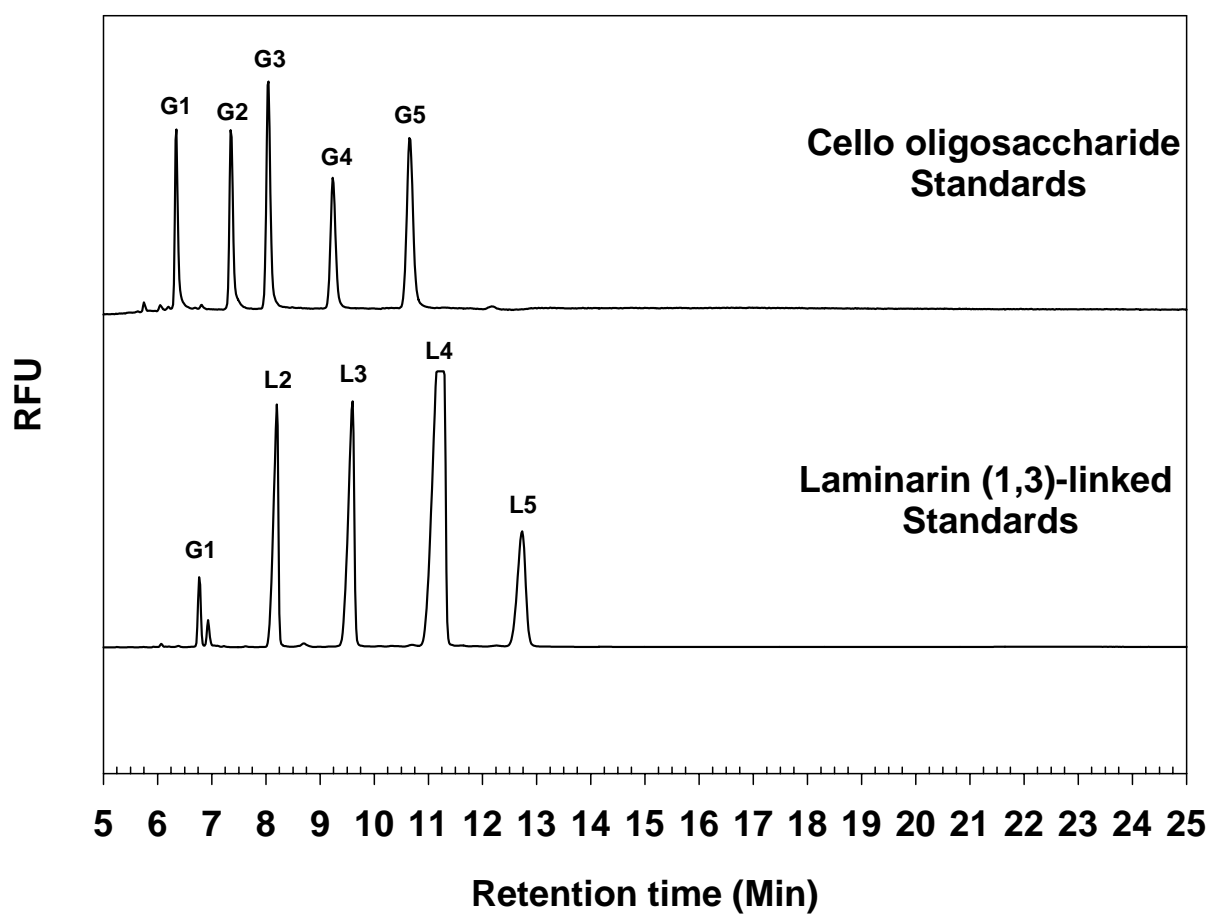

Figure 4A: Laminarin standards ranging from $\mathrm{Glc}_{2}$ to $\mathrm{Glc}_{5}$ were derivatized with APTS and analyzed. These laminarin standards are comprised of $\beta$-(1,3)-linked glucans. When compared with $\beta$ - $(1,4)$-linked cello oligosaccharide standards (Glc to Gl $_{5}$ ) the relative retention times of the $\beta$-(1,3)-linked glucans were offset by an additional two minutes. This factor of increased retention time may be responsible for the altered retention time seen in the $\mathrm{CE}$ analysis of the accumulated glucan intermediates of the CelC mutant (Fig.3). 


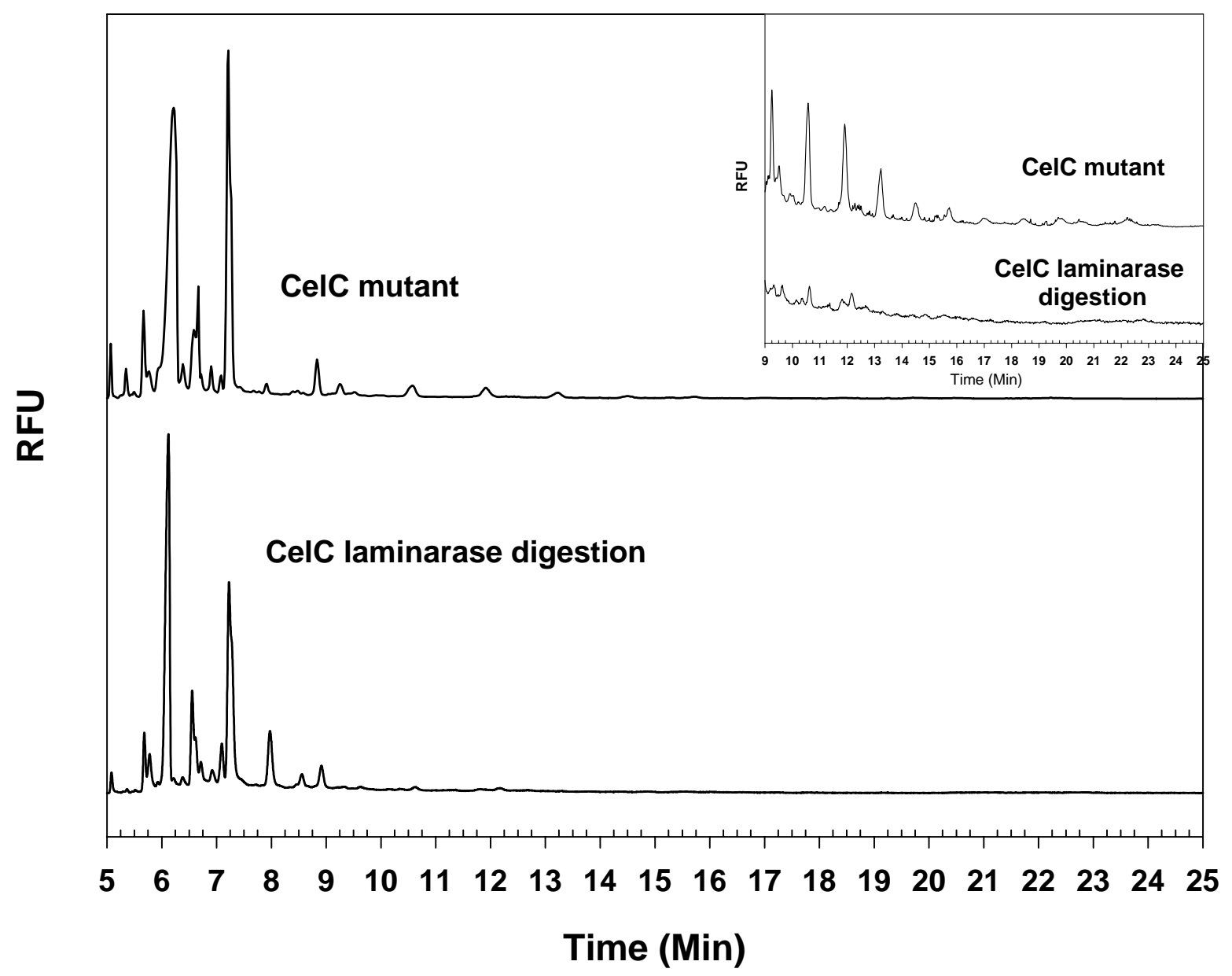

Figure 4B: The CelC mutant intermediates contained a comparatively high proportion of $(1,3)$-linked glucan (37 mol\% of the total glucose present in the mutant) relative to the wild type sample. When these intermediates were subjected to enzymatic digestion by a $\beta-(1,3)$ Laminarinase the novel short chain moieties again disappeared, as with EG-II digestion, which can more clearly be seen in the insert. This data suggests that the intermediates that accumulate in the CelC mutant contain a mixture of $\beta-(1,3)-$ and $\beta-(1,4)$ - linked glucans. 


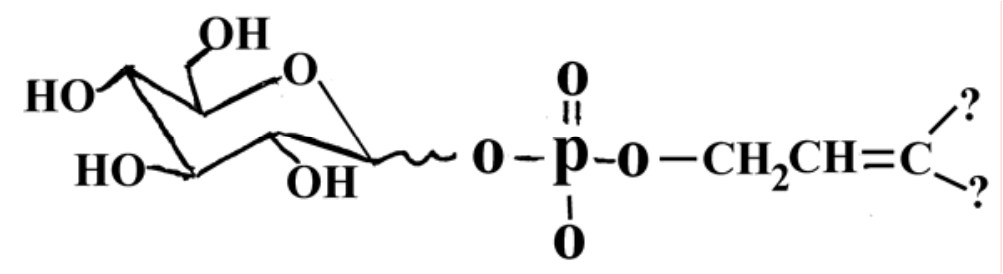

Figure 5: A proposed structure of an allylically-linked glucosyl-1-phospholipid (Chilton and Matthysse, personal communication). It is our belief that the actural lipid constituent of this structure is undecaprenol $\left(\mathrm{C}_{55} \mathrm{H}_{90} \mathrm{O}\right)$. 


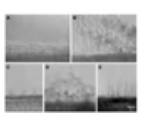

Figure 6: Attachment of A. tumefaciens to roots as viewed in the light microscope with Nomarski optics. Bacteria were incubated with tomato roots for 24 hours at $25^{\circ}$. A. C58 (wild type) shows normal binding. B. C58B224 (CelR mutant) shows much increased binding. C. A1045 (ChvB mutant) shows no binding. D. A1045B224 (ChvB CelR mutant) shows increased binding. E. A1045celAB224 (ChvB CelA CelR mutant) shows no binding. Overproduction of cellulose in CelR mutants leads to increased binding to roots. The celA mutation prevents the synthesis of cellulose and thus the CelA CelR double mutant no longer shows increased binding to roots. Similar results were seen with CelG mutants which also overproduce cellulose. The bar indicates $10 \mu$.

\author{
Reference List
}

Ausmees,N., Jonsson,H., Hoglund,S., Ljunggren,H., and Lindberg,M. (1999) Structural and putative regulatory genes involved in cellulose synthesis in Rhizobium leguminosarum bv. trifolii Microbiology 145 ( Pt 5): 1253-1262.

Matthysse,A.G., Deschet,K., Williams,M., Marry,M., White,A.R., and Smith,W.C. (2004) A functional cellulose synthase from ascidian epidermis Proc.Natl.Acad.Sci.U.S.A 101: 986-991.

Matthysse,A.G., McMahan,S. (1998) Root colonization by Agrobacterium tumefaciens is reduced in cel, attB, attD, and attR mutants Appl.Environ.Microbiol. 64: 2341-2345. 
Matthysse,A.G., Thomas,D.L., and White,A.R. (1995) Mechanism of cellulose synthesis in Agrobacterium tumefaciens J.Bacteriol. 177: 1076-1081.

Matthysse,A.G., White,S., and Lightfoot,R. (1995) Genes required for cellulose synthesis in Agrobacterium tumefaciens J.Bacteriol. 177: 1069-1075.

Romling,U., Bokranz,W., Rabsch,W., Zogaj,X., Nimtz,M., and Tschape,H. (2003) Occurrence and regulation of the multicellular morphotype in Salmonella serovars important in human disease Int.J.Med.Microbiol. 293: 273-285.

Saxena,I.M., Lin,F.C., and Brown,R.M.jr. (1990) Cloning and sequencing of a new gene in an operon for cellulose biosynthesis in Acetobacter xylinum. Plant Mol.Biol. 15: 673-683.

Standal,R., Iversen,T.G., Coucheron,D.H., Fjaervik,E., Blatny,J.M., and Valla,S. (1994) A new gene required for cellulose production and a gene encoding cellulolytic activity in Acetobacter xylinum are colocalized with the bcs operon J.Bacteriol. 176: 665-672.

Stasinopoulos,S.J., Fisher,P.R., Stone,B.A., and Stanisich,V.A. (1999) Detection of two loci involved in (1-->3)-betaglucan (curdlan) biosynthesis by Agrobacterium sp. ATCC31749, and comparative sequence analysis of the putative curdlan synthase gene Glycobiology 9: 31-41.

Weinhouse,H., Sapir,S., Amikam,D., Shilo,Y., Volman,G., Ohana,P., and Benziman,M. (1997) c-di-GMP-binding protein, a new factor regulating cellulose synthesis in Acetobacter xylinum FEBS Lett. 416: 207-211.

Zogaj,X., Bokranz,W., Nimtz,M., and Romling,U. (2003) Production of cellulose and curli fimbriae by members of the family Enterobacteriaceae isolated from the human gastrointestinal tract Infect.Immun. 71: 4151-4158. 